\section{The link between hyperoxia, delayed cerebral ischaemia and poor outcome after aneurysmal SAH: association or therapeutic endeavour}

\author{
Robert M Starke, Neal F Kassell
}

In this study, Jeon et $a l^{1}$ assess the association between exposure to hyperoxia in ventilated patients following aneurysmal subarachnoid haemorrhage (SAH) and both delayed cerebral ischaemia (DCI) and functional outcome. Prospective data from an observational cohort of patients with available partial pressure of oxygen $\left(\mathrm{PaO}_{2}\right)$ was retrospectively assessed. Hyperoxia was defined as the highest quartile of $\mathrm{PaO}_{2}$ $(\geq 173 \mathrm{~mm} \mathrm{Hg})$ and poor outcome as modified Rankin scale 4-6 three months after SAH. Of 252 included patients, there were no significant differences in baseline characteristics between patients with and without a history of hyperoxia. DCI occurred in $38.5 \%$ of patients and unfavourable outcome occurred in 53.5\%. After adjusting for modified Fisher scale, rebleeding, global cerebral oedema, intracranial pressure crisis, pneumonia and sepsis, hyperoxia was independently associated with DCI. After adjusting for age, Hunt-Hess grade, aneurysm size, Acute Physiology and Chronic Health Evaluation II score, rebleeding, pneumonia and sepsis, hyperoxia was independently associated with poor outcome.

The authors should be commended for their study that demonstrates that hyperoxia exposure is associated with worse outcomes. Similarly, studies of patients following cardiac arrest and traumatic brain injury have suggested that hyperoxia is associated with worse outcomes. ${ }^{2} 3$

Department of Neurological Surgery, University of Virginia, Charlottesville, Virginia, USA

Correspondence to Dr Robert M Starke, Department of Neurological Surgery, University of Virginia, P.O. Box 800212, Charlottesville, VA 22908, USA; rms6bx@hscmail.mcc.virginia.edu
Following cerebral injury, hyperoxia may contribute to poor outcomes through a number of mechanisms. ${ }^{4}$ Hyperoxia is postulated to contribute to cerebral vasospasm and DCI through reduction in cerebral blood flow. The present authors found that hyperoxia was associated with poor outcome independently of carbon dioxide levels. Additionally, recent studies provide evidence that local inflammation and cellular injury may result in DCI independently of cerebral vessel diameter alteration. Hyperoxia can increase free radical production, which results in oxidative stress when there is overproduction and/or decreased clearance of free radicals. This can directly lead to cellular toxicity, DNA damage and apoptosis. This contributes to endothelial dysfunction, immune cell localisation and smooth muscle cell migration and proliferation, which further promote vascular inflammation and injury. Hyperoxia may also activate the coagulation cascade with alteration in fibrinolysis. Ultimately, hyperoxia may result in DCI through inflammation, vasoconstriction and microcirculation dysfunction. Thus, exposure to excess oxygen may represent a modifiable factor for morbidity and mortality in patients with SAH.

Conversely, studies have also found that hyperoxia may be of therapeutic benefit and improve outcomes for patients with traumatic brain injury and ischaemic stroke. ${ }^{5}{ }^{6}$ Specifically, patients that are hypoxic and receive appropriate therapeutic supplemental oxygen may have improved outcomes. Patients that have appropriate oxygen levels but are exposed to supra-physiologic oxygen concentration or are treated with higher prophylactic oxygen concentrations may have worse outcomes. Thus, it is unclear whether hyperoxia is associated with poor outcome or is a modifiable risk factor or therapeutic measure. Currently, there are no evidencebased guidelines to guide optimal oxygenation algorithms in patients with $\mathrm{SAH}$ other than the clear relationship with prolonged hypoxia and neurological injury. Going forward, it will be important to determine whether hyperoxia is an association with poor outcome or whether alterations in $\mathrm{PaO}_{2}$ can improve outcome.

Competing interests None.

Provenance and peer review Commissioned; internally peer reviewed.

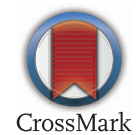

To cite Starke RM, Kassell NF. I Neurol Neurosurg Psychiatry 2014;85:1292.

Received 5 May 2014

Accepted 6 May 2014

Published Online First 29 May 2014

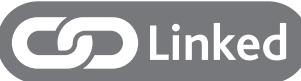

- http://dx.doi.org/10.1136/jnnp-2013-307314

J Neurol Neurosurg Psychiatry 2014;85:1292.

doi:10.1136/jnnp-2014-308326

\section{REFERENCES}

1 Jeon S, Choi A, Badjatia N, et al. Hyperoxia may be related to delated cerebral ischemia and poor outcome after subarachnoid hemorrhage. J Neurol Neurosurg Psychiatry 2014;85:1301-7.

2 Brenner M, Stein D, Hu P, et al. Association between early hyperoxia and worse outcomes after traumatic brain injury. Arch Surg 2012;147:1042-6.

3 Kilgannon $\mathrm{JH}$, Jones $\mathrm{AE}$, Shapiro $\mathrm{NI}$, et al. Association between arterial hyperoxia following resuscitation from cardiac arrest and in-hospital mortality. JAMA 2010;303:2165-71.

4 Dumont AS, Dumont RJ, Chow MM, et al. Cerebral vasospasm after subarachnoid hemorrhage: putative role of inflammation. Neurosurgery 2003;53:123-33; discussion 133-125.

5 Singhal $A B$, Benner $T$, Roccatagliata $L$, et al. A pilot study of normobaric oxygen therapy in acute ischemic stroke. Stroke 2005;36:797-802.

6 Tolias CM, Reinert M, Seiler R, et al. Normobaric hyperoxia-induced improvement in cerebral metabolism and reduction in intracranial pressure in patients with severe head injury: a prospective historical cohort-matched study. J Neurosurg 2004;101:435-44. 